\title{
Integrative analysis of DNA methylation in discordant twins unveils distinct architectures of systemic sclerosis subsets
}

Paula S. Ramos ${ }^{1,2}$ D, Kip D. Zimmerman ${ }^{3,4}$, Sandra Haddad ${ }^{5}$, Carl D. Langefeld ${ }^{3,4}$, Thomas A. Medsger Jr ${ }^{6}$ and Carol A. Feghali-Bostwick ${ }^{1 *}$

\begin{abstract}
Background: Systemic sclerosis (SSc) is a rare autoimmune fibrosing disease with an incompletely understood genetic and non-genetic etiology. Defining its etiology is important to allow the development of effective predictive, preventative, and therapeutic strategies. We conducted this epigenomic study to investigate the contributions of DNA methylation to the etiology of SSc while minimizing confounding due to genetic heterogeneity.
\end{abstract}

Methods: Genomic methylation in whole blood from 27 twin pairs discordant for SSC was assayed over $450 \mathrm{~K} \mathrm{CpG}$ sites. In silico integration with reported differentially methylated cytosines, differentially expressed genes, and regulatory annotation was conducted to validate and interpret the results.

Results: A total of 153 unique cytosines in limited cutaneous SSC (ICSSC) and 266 distinct sites in diffuse cutaneous SSc (dcSSc) showed suggestive differential methylation levels in affected twins. Integration with available data revealed 76 CpGs that were also differentially methylated in blood cells from lupus patients, suggesting their role as potential epigenetic blood biomarkers of autoimmunity. It also revealed 27 genes with concomitant differential expression in blood from SSC patients, including IFI44L and RSAD2. Regulatory annotation revealed that dcSSCassociated CpGs (but not ICSSC) are enriched at Encyclopedia of DNA Elements-, Roadmap-, and BLUEPRINT-derived regulatory regions, supporting their potential role in disease presentation. Notably, the predominant enrichment of regulatory regions in monocytes and macrophages is consistent with the role of these cells in fibrosis, suggesting that the observed cellular dysregulation might be, at least partly, due to altered epigenetic mechanisms of these cells in dcSSc.

Conclusions: These data implicate epigenetic changes in the pathogenesis of SSc and suggest functional mechanisms in SSc etiology.

Keywords: Systemic sclerosis, DNA methylation, Genome, Blood, Twins

\section{Background}

Systemic sclerosis (SSc or scleroderma) is a rare multisystem, connective tissue disease characterized by cutaneous and visceral fibrosis, immune dysregulation, and vasculopathy. Patients are commonly classified into two main clinical subsets on the basis of the extent of skin thickening: limited or restricted cutaneous SSc (lcSSc)

\footnotetext{
*Correspondence: feghalib@musc.edu

${ }^{1}$ Division of Rheumatology and Immunology, Department of Medicine,

Medical University of South Carolina, Charleston, SC, USA

Full list of author information is available at the end of the article
}

and diffuse or widespread cutaneous SSc (dcSSc). The etiology of SSc remains elusive. The low concordance rate in monozygotic twins and relatively modest genetic burden suggest a substantial role for epigenetic or environmental factors in SSc susceptibility [1, 2]. Environmental factors (e.g., nutrition, behavior, stress) can influence methylation and other epigenetic marks that result in phenotypic change and disease [3]. Thus, epigenetic variation may play an important role in SSc risk.

DNA methylation is a chemical modification of cytosine bases generally associated with transcriptional 
repression when at regulatory elements such as promoters and enhancers $[4,5]$. Nevertheless, the precise relationships between DNA methylation and gene expression are complex and poorly understood [5-8]. The correlation between DNA methylation and gene expression can be positive or negative and is tissue-specific and context-specific, in that the local DNA sequence and genomic features largely account for local patterns of methylation [4, 9-11]. In addition to its potential to affect an individual's susceptibility to SSc, changes in the methylation of DNA may occur secondarily to SSc and may consequently influence disease progression. There is compelling evidence that DNA methylation plays a role in the pathogenesis of autoimmune diseases, and multiple epigenome-wide association studies revealed the existence of differentially methylated regions associated with, for example, systemic lupus erythematosus (SLE) [12-17], rheumatoid arthritis [18-26], or psoriasis [27-32]. In SSc, differentially methylated genes were reported in an X chromosome analysis of peripheral blood mononuclear cells [33] and in one genome-wide DNA methylation analysis in dermal fibroblasts [34]. Disease-discordant monozygotic twins offer the ideal study design to investigate the association of DNA methylation with a disease, as it minimizes confounding due to genetic heterogeneity, sex-, age- and early-life environmental effects $[35,36]$.

To our knowledge, no genome-wide investigation of DNA methylation in whole blood from discordant twins has been reported in SSc. We first conducted epigenomic profiling to investigate the association between DNA methylation variation and SSc. Next, we conducted tissue-specific regulatory annotation and integration with available data from DNA methylation and gene expression profiling studies, with the goal of gaining insights into the potential molecular mechanisms underlying SSc development and/or progression.

\section{Methods}

\section{Subjects}

A total of 27 twin pairs discordant for SSc were used for this study (Table 1). All subjects have been previously described in detail [2]. As reported [2], patients were classified based on published criteria [37]. Both twins had to be living to participate in the study. Only samples of self-reported European ancestry were used for this study. The majority of twin pairs were female $(n=26$, $96 \%)$, and approximately two thirds $(n=19,70 \%)$ were monozygotic. The mean age of diagnosis was 43 years, and average disease duration from disease onset (first symptom attributable to SSc) was 8.8 years. We had 17 twin pairs with complete organ involvement data. Among these, the most frequent organ system involvement consisted of Raynaud's phenomenon in 17 twin
Table 1 Characteristics of the twin pairs discordant for SSc used for this analysis

\begin{tabular}{|c|c|c|c|c|c|}
\hline Pair & Zygosity & Subtype & Gender & Autoantibody & Organ involvement \\
\hline 1 & $M Z$ & dcSSc & $\mathrm{F} / \mathrm{F}$ & RNA pol & $\mathrm{JT}, \mathrm{Gl}, \mathrm{DU}, \mathrm{RN}$ \\
\hline 2 & $M Z$ & deSSc & $F / F$ & ATA & $J T, G l, D U$ \\
\hline 3 & $M Z$ & dcSSc & $F / F$ & RNA pol & JT \\
\hline 4 & $M Z$ & deSSc & $F / F$ & RNA pol & $J T, L N, R N$ \\
\hline 5 & $M Z$ & deSSc & $F / F$ & U3 & JT \\
\hline 6 & DZ & deSSc & $F / F$ & RNA pol & JT, Gl, DU \\
\hline 7 & $M Z$ & ICSSC & $F / F$ & U1 & $\mathrm{Gl}$ \\
\hline 8 & $M Z$ & ICSSC & $F / F$ & PL-7 & $J T, G l, L N$ \\
\hline 9 & $M Z$ & ICSSC & $\mathrm{F} / \mathrm{F}$ & U3 & $\mathrm{JT}, \mathrm{Gl}$ \\
\hline 10 & $\mathrm{DZ}$ & ICSSC & $F / F$ & ACA & $J T, D U$ \\
\hline 11 & DZ & dcSSc & $\mathrm{F} / \mathrm{F}$ & ATA & JT, Gl, DU, LN \\
\hline 12 & $M Z$ & $\mathrm{dcSSC}$ & $F / F$ & Unknown & JT, Gl, LN \\
\hline 13 & $M Z$ & dcSSc & $F / F$ & RNA pol & N/A \\
\hline 14 & DZ & ICSSC & $F / F$ & ACA & JT \\
\hline 15 & DZ & ICSSC & $F / F$ & RNA pol & N/A \\
\hline 16 & DZ & ICSSC & $\mathrm{F} / \mathrm{M}$ & ATA & $\mathrm{N} / \mathrm{A}$ \\
\hline 17 & DZ & ICSSC & $F / F$ & PM-Scl & $J T, G l$ \\
\hline 18 & DZ & ICSSC & $F / F$ & U1 & JT \\
\hline 19 & $M Z$ & ICSSC & $F / F$ & $A C A$ & JT, Gl, DU \\
\hline 20 & $M Z$ & ICSSC & $F / F$ & U3 & $\mathrm{Gl}, \mathrm{DU}$ \\
\hline 21 & $M Z$ & ICSSC & $F / F$ & ATA & $\mathrm{N} / \mathrm{A}$ \\
\hline 22 & $M Z$ & ICSSC & $\mathrm{M} / \mathrm{M}$ & RNA pol & N/A \\
\hline 23 & $M Z$ & ICSSC & $F / F$ & ACA & N/A \\
\hline 24 & $M Z$ & ICSSC & $F / F$ & ACA & $\mathrm{N} / \mathrm{A}$ \\
\hline 25 & $M Z$ & ICSSC & $F / F$ & ACA & N/A \\
\hline 26 & $M Z$ & ICSSC & $F / F$ & $A C A$ & N/A \\
\hline 27 & $M Z$ & ICSSC & $F / F$ & U1 & N/A \\
\hline
\end{tabular}

$M Z$ monozygotic, $D Z$ dizygotic, $F$ female, $M$ male, $A C A$ anticentromere, $R N A$ pol anti-RNA polymerases, ATA anti-topoisomerase I, U1 anti-U1 RNP, U3 anti-U3 RNP, PL-7 anti-PL-7, PM-SCl anti-PM-Scl, Unknown autoantibodies did not recognize known autoantigens $J T$ joint or tendon $G /$ gastrointestinal, $D U$ digital ulcers, $L N$ lung, $R N$ renal, $N / A$ data not available

pairs $(100 \%)$, joint or tendon in $15(88 \%)$, gastrointestinal in 11 (65\%), digital ulcers in 7 (41\%), lung in 4 (24\%), and renal involvement in 2 (12\%). Among the 27 patients, the most common SSc-associated serum autoantibodies were anticentromere $(n=7,26 \%)$, anti-RNA polymerases $(\mathrm{n}=7,26 \%)$, anti-topoisomerase I $(n=4$, $15 \%)$, anti-U1 RNP $(n=3,11 \%)$, and anti-U3 RNP $(n=$ 3, 11\%). For the disease subset analyses, 15 pairs with lcSSc and 9 pairs with dcSSc were used. Genomic DNA was extracted from whole blood from all 27 pairs of twins as previously described [2].

\section{Zygosity testing}

Twin zygosity was initially assayed using DNA fingerprint analysis as we described [2]. In addition, zygosity 
was confirmed by the analysis of 11 short tandem repeat (STR) autosomal markers using the GenomeLab Human STR Primer Set kit on a CEQ8000 Genetic Analysis System (Beckman Coulter, Fullerton, CA) or 15 autosomal STR markers using the AmpFLSTR Identifiler PCR Amplification Kit on a 3500 Genetic Analyzer (Applied Biosystems, Foster City, CA). The manufacturer's protocols were followed for both systems with one exception: separations on the 3500 Genetic Analyzer were performed with POP7 on a 50-cm array.

\section{DNA methylation assay and data analysis}

Genomic DNA $(1 \mu \mathrm{g})$ from each individual was treated with sodium bisulfite using the EZ 96-DNA methylation kit (Zymo Research, USA), following the manufacturer's standard protocol. Genome-wide DNA methylation was assessed in the Genomics Research Core at the University of Pittsburgh using the Illumina Infinium HumanMethylation450 BeadChip (Illumina, USA), which interrogates over 485,500 CpG sites that cover $99 \%$ of RefSeq genes (including the promoter, 5' UTR, first exon, gene body, and 3'UTR), as well as 96\% of CpG islands and island shores. Arrays were processed using the manufacturer's standard protocol. Location of individuals on arrays was randomized to minimize potential confounding (e.g., batch effects).

Sample files and expression IDAT files were imported into GenomeStudio Software v.1.9 (Illumina, USA) for primary evaluation of the data. This included initial quality control checks and calculating the relative methylation level of each interrogated cytosine, which is reported as a $\beta$-value given by the ratio of the normalized signal from the methylated probe to the sum of the normalized signals of the methylated and unmethylated probes. A negative $\beta$-value indicates hypomethylation (i.e., decreased methylation) in the affected SSc twins relative to the unaffected, while a positive $\beta$-value indicates hypermethylation (i.e., increased methylation) in the SSc twins relative to the unaffected twins. The data were observed for quality, and a cluster analysis was conducted, using the SNP content, to ensure twins were pairing correctly. Using GenomeStudio, it was noted that the data contained no large batch effects.

After initially inspecting the data with GenomeStudio, the data was opened with the $R$ package ChAMP [38]. When loading the data, probes were dropped if they had a bead count less than 3 , if the probed CpG was also an SNP, or if they did not meet a detection $p$ value of $1 \times 10^{-5}$ (detection $\mathrm{p}$-value is the confidence that a given transcript is expressed above the background defined by negative probes). A total of 447,254 CpGs were used for analysis. The data were then normalized with the same ChAMP package using a BMIQ normalization method. MDS plots based on the 1000 most variable methylation sites were created as a result of the normalization process. These were examined for clustering, and it appeared that samples from individuals of differing ethnicities were clustering together, so some samples were removed to make a more homogenous group that clustered closely together. Singular value decomposition (SVD) was then applied to the matrix to obtain the most significant components of variation. These components were observed in a heat map showing the association between the principal components and the biological factors. To adjust for these batch effects, the ChAMP package employs "ComBat" which uses empirical Bayes methods to correct for technical variation. With the data normalized and batch effects adjusted for, the $\beta$-values were outputted to a table for analysis.

A paired $t$ test was computed for each CpG site to test the null hypothesis that the mean difference of $\beta$-values for each set of twins is zero $(\mu=0)$. Completing a matched analysis with the paired $t$ test allows us to remove the confounding effects of chronological age, genetic background, ethnicity and admixture, sex, and similarity of the epigenome at birth. All data for monozygotic twins $(n=19)$ were analyzed first followed by a replication of that analysis with the data for dizygotic twins $(n=8)$. A meta-analysis of the two separate analyses was then performed using METAL [39] to get a single $p$ value for each $\mathrm{CpG}$ site. False discovery rate (FDR) $p$ values were then calculated for each site, and top results were evaluated. Since no differentially methylated cytosine was identified with FDR-corrected $p<$ 0.05 , unadjusted $p$ values are reported. Only cytosines showing suggestive differential methylation $\left(p<10^{-04}\right)$ in the meta-analysis between the affected and unaffected twin pairs are reported.

Monozygotic twins exhibit increased DNA methylation differences with age [40]. In order to address the effect of age on DNA methylation variation in this study, we cross-referenced our results (all cytosines with suggestive differential methylation $\left(p<10^{-04}\right)$ ) against the 490 and the 353 differentially methylated CpG sites associated with age reported by Bell et al. [41] and Horvath [42], respectively.

Despite the limited statistical power, an exploratory analysis was computed comparing all twins positive for each of the following clinical features: (1) lung involvement, (2) anticentromere autoantibodies (ACA), and (3) anti-RNA polymerases autoantibodies (anti-RNP), to the twins negative for these criteria. No CpGs met the threshold for suggestive differential methylation $\left(p<10^{-04}\right)$ for any of these clinical features. 


\section{Pathway analysis}

Ingenuity Pathway Analysis (IPA) software (https://www. qiagenbioinformatics.com/products/ingenuity-pathwayanalysis/) was used (release date 16 March 2016) to investigate the pathways and functions enriched with the molecules corresponding to the top differentially methylated genes. IPA uses an extensive database of functional interactions that are drawn from peer-reviewed publications and manually maintained. Core Analyses were performed using default settings to identify the top canonical pathways, diseases and biological functions, physiological systems, networks, and upstream regulators. For each comparison of the DNA methylation analyses, a total of 200 molecules (i.e., gene products) corresponding to the top differentially methylated cytosines were used as input for IPA Core Analyses. Specifically, the top 200 genes corresponding to the top differentially methylated cytosines were used as input into IPA's Core Analysis; the products of these genes were used by IPA as molecules to predict, for example, downstream biological processes or diseases affected by the data, or upstream molecules which may be causing the observed changes in the data.

\section{Regulatory annotation}

eFORGE v1.2 (http://eforge.cs.ucl.ac.uk/) [43] was used to identify if the associated CpGs were enriched in cell-specific regulatory elements, namely DNase I hypersensitive sites (DHSs) (markers of active regulatory regions) and loci with overlapping histone modifications (H3Kme1, H3Kme4, H3K9me3, H3K27me3, and H3K36me3) across available cell lines and tissues from the Roadmap Epigenomics Project, BLUEPRINT Epigenome, and ENCODE (Encyclopedia of DNA Elements) consortia data. In addition to predicting disease-relevant cell types, eFORGE can also assess cell-composition effects of heterogeneous tissues by detecting tissue-specific DHS and histone modification enrichment based on genomic location.

The differentially methylated cytosines $\left(p<10^{-04}\right)$ in each disease subset (153 in lcSSc, 266 in dcSSc, and 155 in all twins) were entered as input of the eFORGE analysis (Additional file 2). Each set of CpGs was tested for enrichment for overlap with putative functional elements compared to matched background CpGs. The matched background is a set of the same number of CpGs as the test set, matched for gene relationship and $\mathrm{CpG}$ island relationship annotation. One thousand matched background sets were applied. The enrichment analysis was completed for different tissues, since functional elements may differ across tissues. Enrichment outside the 99.9th percentile $\left(-\log _{10}\right.$ binomial $p$-value $\left.\geq 3.38\right)$ was considered statistically significant (red in Additional file 2: Figure S2, Figs. 1 and 2).

\section{Results}

Differentially methylated sites in whole blood from twins discordant for SSC

We performed genome-wide DNA methylation analysis in whole blood from 27 twin pairs discordant for SSc (Table 1). Monozygotic twins $(n=19)$ were analyzed first, followed by a replication with the data for dizygotic twins $(n=8)$. This manuscript reports the results of the meta-analysis of this discovery and replication sets. A total of 155 cytosines showed suggestive differential methylation $\left(p<10^{-04}\right)$ between the affected and unaffected twin pairs, most of which mapped to gene bodies $(113,73 \%)$ of 111 unique genes (Additional file 2: Table S1). We note that while a negative $\beta$-value $(-1<\beta$ $<0)$ indicates hypomethylation (i.e., decreased methylation) in the affected SSc twins relative to the unaffected, a positive $\beta$-value $(0<\beta<1)$ indicates hypermethylation (i.e., increased methylation) in the SSc relative to the unaffected twins. Results in monozygotic and dizygotic twin pairs were largely consistent (Additional file 2: Table S1). The levels of differential methylation between affected and unaffected twin were overall modest, with the largest difference observed in the IFI44L gene $(\beta$-value $=-0.12)$ (Additional file 1: Figure S1). Pathway analysis revealed a significant enrichment of molecules (i.e., gene products) involved in cancer, gastrointestinal disease, and organismal injury and abnormalities (Additional file 2: Table S2).

We also performed DNA methylation analyses in each disease subset. In the meta-analysis of 15 twin pairs discordant for lcSSc, 153 cytosines showed suggestive differential methylation $\left(p<10^{-04}\right)$ between the affected and unaffected twin pairs, most of which mapped to gene bodies $(117,77 \%)$ of 115 distinct genes (Additional file 2: Table S3). The differences of methylation levels were modest ( $\beta<$ $0.10)$. Pathway analysis showed a significant enrichment of cancer, endocrine system disorders, gastrointestinal disease, and organismal injury and abnormalities (Additional file 2: Table S4). A total of 266 cytosines showed suggestive (meta-analysis $p<10^{-04}$ ) differential methylation levels in whole blood from the 9 pairs of twins discordant for dcSSc. The majority of these cytosines mapped to gene bodies (201, 76\%) of 196 distinct genes (Additional file 2: Table S5). The largest differences in methylation levels were observed in the hypomethylated IFI44L $(\beta=-0.17)$ and DHODH $(\beta=-0.17)$ genes. The top molecules were enriched for cancer, gastrointestinal disease, and organismal injury and abnormalities (Additional file 2: Table S6). While there was virtually no overlap of molecules between subsets, with only $1 \%$ of common molecules (3/397), there was similar overall enrichment for genes in "cancer" and "gastrointestinal disease."

Despite the limited statistical power, an exploratory case-case analysis was computed on the following 
clinical features: (1) lung involvement, (2) anticentromere autoantibodies (ACA), and (3) anti-RNA polymerases autoantibodies (anti-RNP). No CpGs met the threshold for suggestive differential methylation $(p<$ $10^{-04}$ ) for any of these clinical features.

\section{Overlap of DNA methylation patterns with reported genetic association and DNA methylation studies}

We assessed the overlap between the regions our study unveiled (meta-analysis $p<10^{-04}$ ) and over 40 regions with compelling evidence for genetic association with SSc [1]. The few regions of overlap include the $H L A$ and IRF5 (Additional file 2: Table S7). Since aging can influence DNA methylation variation, we also assessed the overlap between our results and the CpG sites whose methylation levels are strongly correlated with chronological age [42, 44]. Only one age-associated CpG (cg22432269) in the first exon of the CYFIP1 gene showed concomitant evidence of hypomethylation in $\operatorname{lcSSc}\left(p=3.06 \times 10^{-06}\right)$.

One genome-wide DNA methylation study has been reported in cultured dermal fibroblasts from SSc patients and controls [34]. As expected, given the different tissues profiled, the genes identified in each study are largely different. Of the 30 genes reported by Altorok et al [34] as common between dcSSc and lcSSc fibroblasts, only CACNA1C was also found among our top results (Additional file 2: Table S2). Additional file 2: Table S8 shows the six CpG sites common to both studies.

Since SSc and SLE are often considered related diseases, we also compared our DNA methylation findings in blood from SSc patients to cytosines reported as differentially methylated in blood (and blood cells) from SLE patients. These included 86 cytosines in naïve CD4 + T cells [45], 1082 CpGs in T cells, 264 CpGs in B cells, 168 CpGs in monocytes [15], 293 sites in neutrophils [46], 26,298 CpGs in PBMCs [47], and 44 cytosines differentially methylated in white blood cells from SLE patients [48]. A total of 76 CpGs differentially methylated in blood cells from both SSc and SLE patients are shown in Additional file 2: Table S9. Several cytosines were reported in multiple studies, notably those differentially methylated in the dcSSc subset, as well as all twins. CpG sites in the IFI44L and RSAD2 genes were consistently hypomethylated in several blood cell types [15, 45-48] or hypermethylated in the case of FNBP1 [15, 47]. In the dcSSc subset, CpG sites in IRF5, INTS6, SULT1A1, and RPTOR were also hypomethylated in multiple blood subsets $[15,46,47]$. These differentially methylated sites shared in blood cells across related autoimmune diseases suggest their role as potential susceptibility or epigenetic blood biomarkers of autoimmunity.

\section{Comparison of differential methylation to differential gene expression patterns}

To explore the downstream effects of the differentially methylated CpG sites (meta-analysis $p<10^{-04}$ ), the genes corresponding to these $\mathrm{CpGs}$ were compared to available data from published global gene expression profiling studies conducted in blood and its cellular subsets from SSc patients and healthy controls. A total of 1907 unique differentially expressed genes were compiled from 8 studies with publicly available results [4956]. As shown in Table 2, 27 genes with differentially methylated cytosines (in Additional file 2: Tables S1, S3, and S5) have also been reported as differentially expressed in SSc patients. Consistent with the known complex relationships between DNA methylation and gene expression [4-11], for some genes, the relationship between DNA methylation and gene expression was inverse or negative (i.e., increased methylation with decreased gene expression), while for others, it was direct or positive (i.e., increased methylation results in increased gene expression).

Eight noteworthy candidates include IFI44L, where cg03607951 in the transcription start site was hypomethylated in all twins and showed the largest difference in methylation levels in dcSSc. This gene is overexpressed in blood in SSc patients [50, 51, 53] and hypomethylated in multiple blood SLE subsets $[15,45,46,48]$. The TLE3 gene showed two CpGs in the gene body (cg01666796, cg12349571) with consistent hypermethylation in all affected twins and is also overexpressed in PBMCs in SSc-PAH patients [49]. A CpG (cg22432269) in the first exon of the CYFIP1 gene showed the most significant hypomethylation in lcSSc concomitant with underexpression in PBMCs from lcSSc patients [51]. A CpG site (cg06580770) in the body of TNXB is hypermethylated in blood from SSc and concomitantly underexpressed in SSc patients [54]. Hypomethylated cg15346781 in the transcription start site of the RSAD2 gene is overexpressed in SSc $[50,53]$ and hypomethylated in $\mathrm{T}$ and $\mathrm{B}$ cells from SLE patients [15]. Cg24312520 in the gene body of STAT3 was hypermethylated in dcSSc and overexpressed in PBMC from lcSSc and SSc-PAH patients $[49,51]$. A first exon cytosine (cg25330422) was reported as hypermethylated in blood cell subsets from SLE patients [15]. The transcription start site TNFRSF1A was both hypermethylated in dcSSc (cg26254667) and overexpressed in SSc and lcSSc [51, 54]. Other gene body CpG sites (cg08418872, cg23752651) have also been reported as hypermethylated in SLE patients [15]. Lastly, cg17925829 in the transcription start site of the TYROBP gene was hypomethylated and the gene overexpressed in SSc $[53,54]$. 


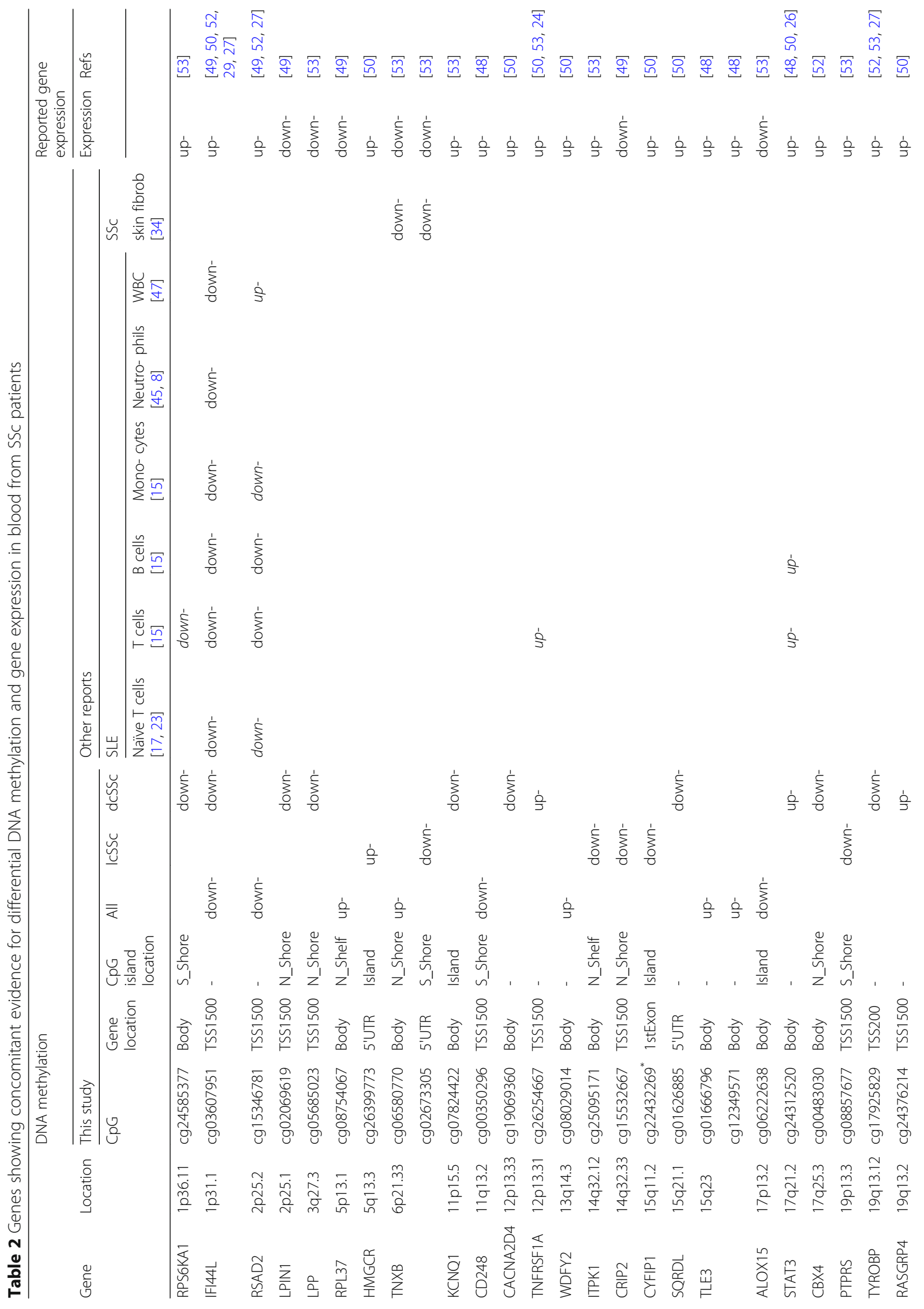




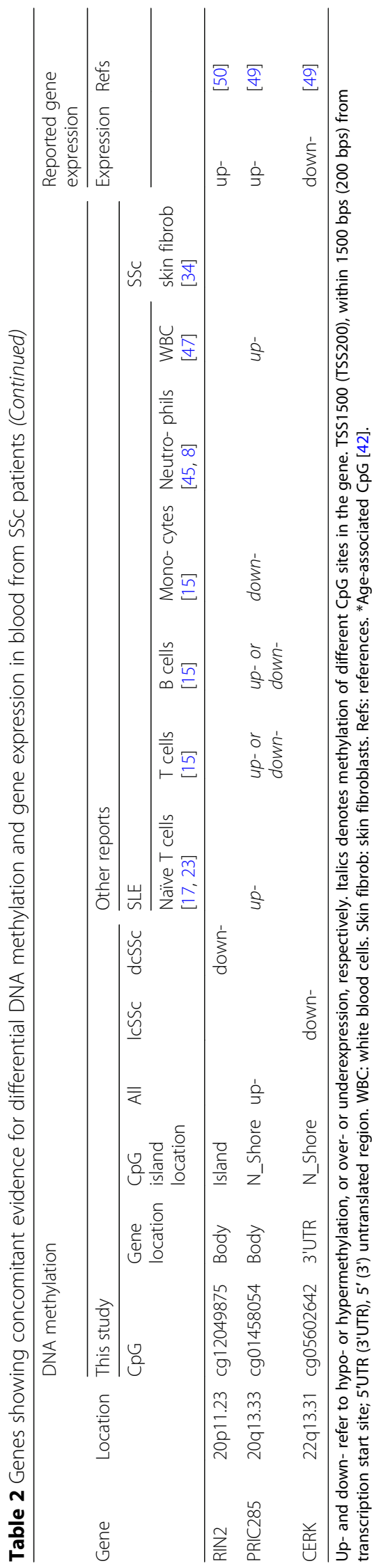




\section{Regulatory annotation}

To provide a broader biological interpretation of the DNA methylation results and better understand the functional role underlying the disease-associated $\mathrm{CpG}$ sites, we assessed whether these SSc-associated CpGs reside within regulatory regions across the genome in diverse tissues and cell types assayed in the ENCODE, Roadmap Epigenomics, and BLUEPRINT Epigenome Project datasets. The CpGs associated with SSc in all twins showed only a modest enrichment of H3K27me3, a mark of inactive genes, in primary B cells (Additional file 1: Figure S2 and Additional file 2: Table S10). The CpGs associated with lcSSc did not show enrichment of either DHSs or any histone mark in any tissue. In contrast, the dcSSc-associated CpGs showed robust enrichment in DHSs across multiple tissues and cell types (Fig. 1; Additional file 2: Table S11). In the ENCODE data, the strongest enrichment was in multiple blood cell lines, predominately myeloid cells, but also the epithelium, heart, muscle, blood vessel, and connective tissue (Fig. 1, top panel; Additional file 2: Table S11). In the Roadmap data, the greatest enrichment of DHS was in the blood, fetal tissues, and psoas muscle (Fig. 1, middle panel; Additional file 2: Table S11). In the hematopoietic primary cells of the BLUEPRINT project, inflammatory macrophages showed strong enrichment of DHSs (Fig. 1, bottom panel; Additional file 2: Table S11). This data provides evidence supporting the notion that CpGs identified in blood are also situated in known active regulatory regions in not only blood, but also other tissues and cell types. Overlapping with $\mathrm{H} 3$ histone methylation from the Roadmap Project revealed that the dcSSc-associated CpGs are strongly enriched for H3K4me1 marks, which are indicative of poised enhancers, across numerous tissues and cell types, most strongly in the blood, fetal tissues, psoas muscle, and skin (Fig. 2, Additional file 2: Table S11). An enrichment of H3K27me3, a mark associated with inactive gene promoters, was also detected in primary hematopoietic stem cells (Fig. 2, Additional file 2: Table S11).

Collectively, this regulatory annotation data shows that, unlike the lcSSc-associated CpGs, many of the dcSSc-associated CpGs reside within DHS and multiple histone marks. This evidence of enrichment of regulatory regions supports their potential role in causal downstream effects on disease presentation.

\section{Discussion}

This study used a genome-wide integrative approach to identify differential DNA methylation in whole blood from twin pairs discordant for SSc. In addition to being the largest epigenomic study conducted in SSc to date, the unique study design minimizes confounding due to genetic heterogeneity and age- and early-life environmental effects by using disease-discordant twins $[35,36]$. As expected, given the sample size, we did not detect genome-wide significant differences in mean DNA methylation associated with SSc, which is largely consistent with other complex disease epigenomic twin studies [12, 57, 58]. The results revealed distinct DNA methylation patterns in SSc and its clinical disease subsets. The negligible overlap of molecules shared between the lcSSc and dcSSc subsets supports distinct epigenetic architectures in each disease subset. Despite clearly distinct blood methylation profiles, an enrichment of genes in "cancer" and "gastrointestinal disease" was observed in both dcSSc and lcSSc, although driven by different molecules. These results are consistent with the previously reported minimal common differentially methylated cytosines between lcSSc and dcSSc subsets in skin fibroblasts [34]. In addition, our analyses revealed negligible overlap between the methylation patterns in whole blood and those previously reported in skin fibroblasts [34]. Thus, although SSc is commonly considered a single disease, these results confirm others suggesting that SSc is a family of diseases with distinctly different subtypes.

The precise relationships between DNA methylation and gene expression are complex and poorly understood [4-11]. While DNA methylation at regulatory elements shows a negative correlation with transcription, the opposite has been observed at intragenic regions [5], illustrating that complex regulatory mechanisms that are dependent on the tissue and genomic architecture underlie the correlation between DNA methylation and gene expression. It is also possible that the low correlation between DNA methylation and gene expression levels may reflect high fluctuation of RNA levels, which can change from $1 \mathrm{~h}$ to the next [59]. In order to provide insights into the potential functional consequences of the methylation patterns observed, we compared our results to those of global gene expression profiling assays conducted in blood and its cellular subsets from SSc patients and healthy controls. This study unveiled several novel genes epigenetically dysregulated with reported changes in gene expression in blood from SSc patients. Most of these genes are involved in immune processes.

$I F I 44 \mathrm{~L}$, an interferon gene involved in defense response to viruses, is overexpressed in SSc blood tissues [50, 51, 53]. The CpG site unveiled in our study shows consistent hypomethylation in multiple blood cell subsets from SLE $[15,45-48,60]$ and Sjögren's syndrome patients $[61,62]$. Since SSc and SLE are often considered as sister diseases, reported DNA methylation similarities are not unexpected [63]. The consistent hypomethylation of IFI44L in blood from patients with several autoimmune diseases, together with its overexpression, corroborates the validity of our finding and suggests that differential methylation 


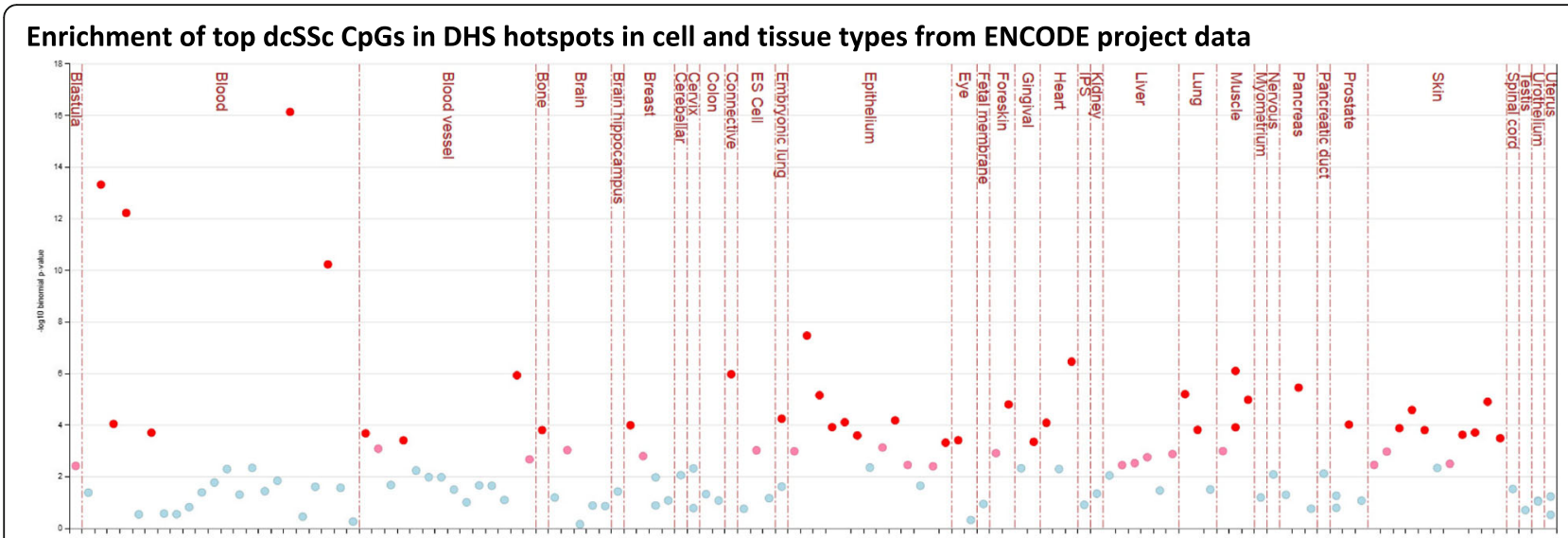

Enrichment of top dcSSc CpGs in DHS hotspots in cell and tissue types from Roadmap Epigenomics project data

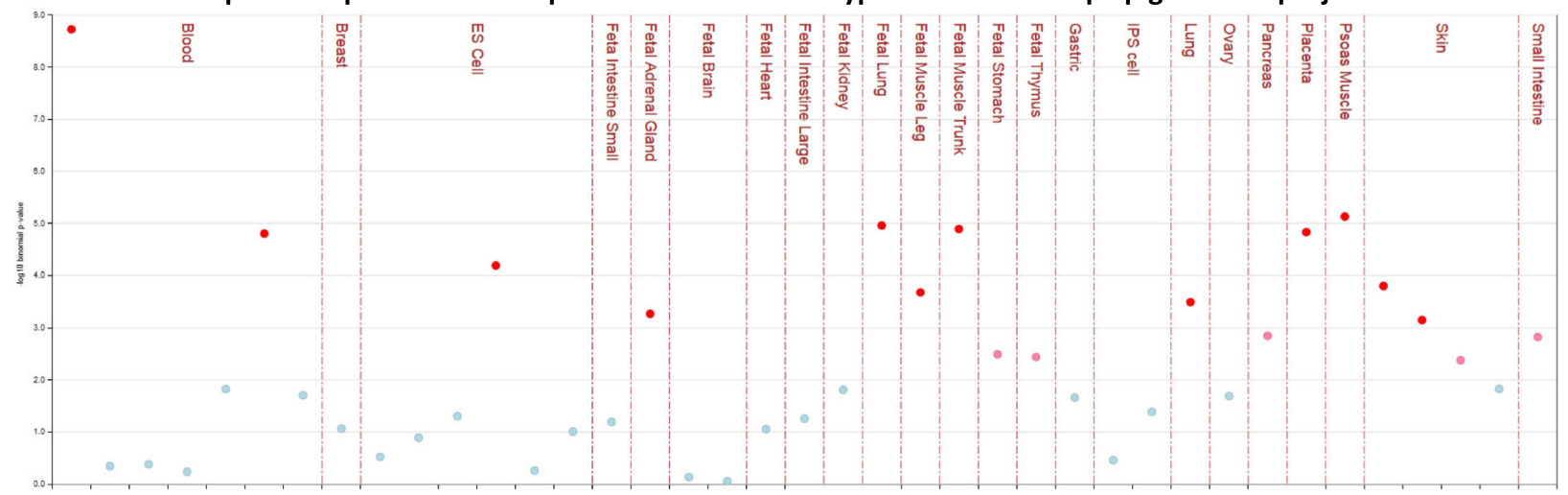

Enrichment of top dcSSc CpGs in DHS hotspots in cell and tissue types from BLUEPRINT Epigenome data.

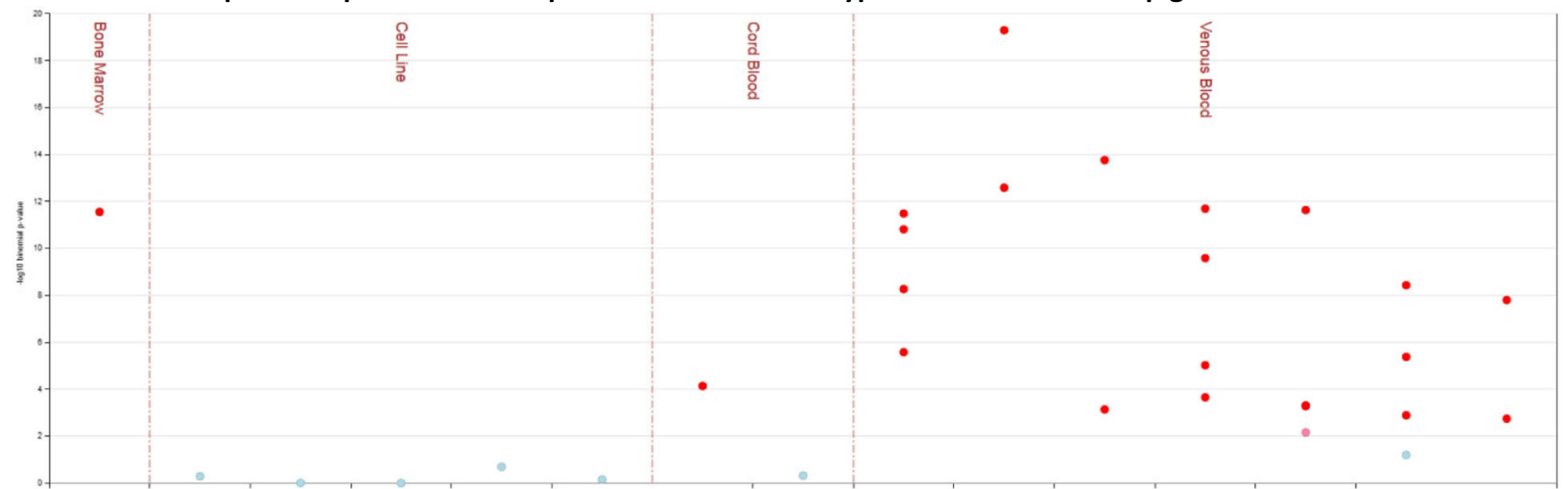

Fig. 1 Enrichment of dcSSC differentially methylated CpGs in DNase I hypersensitive sites among various cell and tissue types using ENCODE, Roadmap Epigenomics, and BLUEPRINT Epigenome projects data. Statistically significant enrichment outside the 99.9th percentile (-log10 binomial $p$ value $\geq 3.38$ ) is colored red on the vertical axis. Upper panel shows a marked myeloid cell enrichment in ENCODE data, with strong epithelium, heart, muscle, blood vessel, and connective tissue signals. Middle panel shows a more general pattern of enrichment, strongest in blood, fetal tissues, and psoas muscle in the Roadmap Epigenomics data. Lower panel shows enrichment for inflammatory macrophages in the BLUEPRINT Epigenome data

of IFI44L may serve as shared biomarker across these diseases.

Both RSAD2 and TYROBP showed hypomethylation and overexpression in SSc blood [50, 53, 54]. Both play roles in immune response, including type I IFN signaling pathway (RSAD2) and innate immunity (TYROBP). $R S A D 2$ is consistently hypomethylated in blood cells [15,
47]. Demethylation of the TYROBP gene is associated with a subset of $\mathrm{T}$ cells that accumulates and is associated with aging [64]. An age-associated CpG [42] in CYFIP1, a regulator of translation and cytoskeletal dynamics, showed hypomethylation with underexpression in SSc blood [51]. It is interesting to note the variation in methylation levels at sites associated with aging, as 


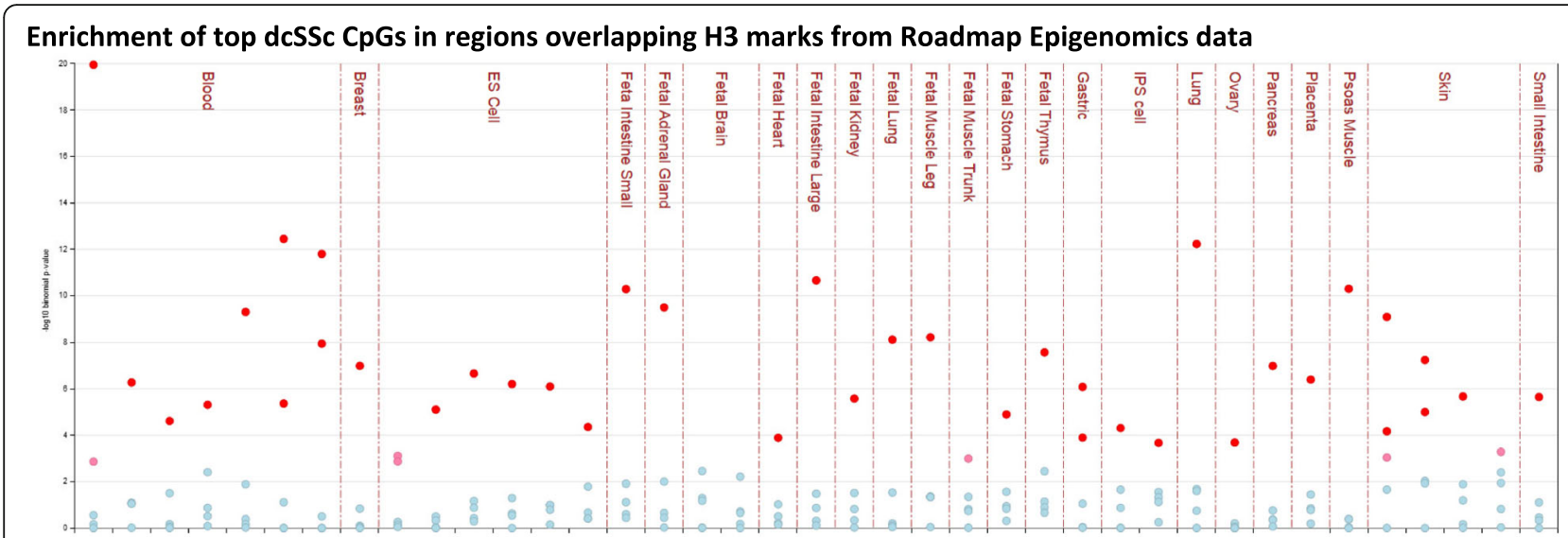

Fig. 2 Enrichment of dcSSC differentially methylated CpGs in regions overlapping histone modifications in the Roadmap Epigenomics Project data. Statistically significant enrichment outside the 99.9th percentile $\left(-\log _{10}\right.$ binomial $p$ value $\left.\geq 3.38\right)$ is colored red on the vertical axis. Panel shows marked enrichment for a histone modification representative of enhancers (H3K4me1) in blood cells (monocytes, hematopoietic stem cells, natural killer cells), fetal tissues (lung fibroblasts, large intestine, small intestine, adrenal gland, muscle leg, thymus), psoas muscle, and skin fibroblasts. Enrichment for a histone modification representative of polycomb-repressed regions (H3K27me3) was seen in hematopoietic stem cells

premature activation of aging-associated molecular mechanisms is emerging as an important contributor to the autoimmune, vascular, and fibrotic pathogenesis of SSc [65]. Our findings, in conjunction with these reports, further lend support for the role of the innate immune response in the pathogenesis and/or progression of diseases such as SSc and a parallel between SSc and premature aging.

Differential methylation of several genes has been reported as associated with cancer [66-69]. These include TNFRSF1A, which plays a role in cell survival, apoptosis, and inflammation and was both hypermethylated and overexpressed in SSc blood [51, 54]. TLE3 was also hypermethylated and overexpressed in SSc blood [49]. This gene product functions in the Notch signaling pathway to regulate the determination of cell fate during development. STAT3, a transcription activator with roles in many cellular processes such as cell growth, apoptosis, and response to cytokines and growth factors, showed hypermethylation and overexpression in SSc blood $[49,51]$. TNXB, which was hypomethylated in skin fibroblasts from dcSSc [34], was hypermethylated in our study and concomitantly underexpressed in blood from SSc patients [54]. This gene localizes to the MHC class III region and encodes a member of the tenascin family of extracellular matrix glycoproteins. It is involved in actin cytoskeleton organization, cell adhesion, and collagen fibril organization.

To aid in result interpretation, regulatory annotation of the top differentially methylated cytosines was conducted to predict disease-relevant cell types. Differential DNA methylations in regulatory regions such as DHS and histone marks have been associated with functional consequences [4, 70]. We observed an enrichment of regulatory regions in the dcSSc subset that pointed to blood myeloid cells as the most highly enriched cell types, indicating a tendency for cell-composition-corrected dcSSc-associated DNA methylation changes to co-locate with myeloid cell DHSs and H3K4me1 marks (representative of enhancers). This contrasts with an enrichment in DHSs specific to T cells that was reported using cytosines differentially methylated in CD4+ T cell studies of SLE and Sjögren's syndrome [43]. This enrichment of methylated cytosines in regulatory regions in myeloid cells might underlie a dysregulation of these cells in dcSSc. Indeed, both monocytes and macrophages (cell types with the strongest enrichment) play a critical role in fibrosis [71]. The number of circulating monocytes is increased in SSc [72] and correlates with disease progression and severity $[73,74]$. The changes in methylation detected in dcSSc are thus impacting the function of regulatory elements in cell types with critical functions in fibrosis. Since these inflammatory cells are dysregulated in SSc, and DNA methylation changes can affect regulatory mechanisms, our findings suggest that DNA methylation might be a potential avenue to reverse their altered phenotype.

This study has a number of limitations. Despite the value of the twin-pair study design for epigenomic studies, our unique samples of middle-aged, European ancestry, largely female twin pairs are not representative of the general population. Thus, our results might not be generalizable to all patients. Further replication studies are warranted for the validation, justification, and generalization of our results. Another limitation is the lack of available RNA from the same samples to assess the functional effects of the variation in DNA 
methylation. In an attempt to circumvent this limitation, we performed in silico integration with reported differentially expressed genes for functional validation of our results. Documenting that differentially methylated sites in our twin data also correspond to differences in gene expression in independent SSc samples forms corroborating evidence across genomic processes and cohorts. A further limitation is the lack of tissue specificity. We explored this issue by performing regulatory annotation of our results, but future work is needed to dissect the tissue specificity of epigenetic modifications in SSc. We cannot exclude the possibility that the differences between the disease subsets and enrichment of myeloid-related cells in dcSSc are driven by confounding cell-composition effects instead of true cell type-specific effects. However, whole blood lymphocytes are proportionally more abundant than monocytes, suggesting that the strong bias towards monocytes and macrophages is a cell type-specific effect. Multiple differentially methylated cytosines in our study were also found to be differentially methylated in a single blood cell type in SLE, suggesting that the associations we detected are not likely to be due to confounding by blood cell heterogeneity. These include, among others, loci in the IFI44L, RSAD2, IRF5, and RPTOR genes [46]. In spite of these limitations, these findings identify novel genomic regions in SSc in a unique cohort of discordant twins and highlight candidate genes for further research.

\section{Conclusions}

We identified multiple DNA methylation loci associated with SSc, including sites with concomitant evidence of altered methylation in blood cells of lupus patients and genes with concomitant evidence of differential expression in blood cells from SSc patients. Although this cross-sectional study cannot separate causality from response to disease, it identifies epigenetically modified genes and pathways that are important in SSc.

Our study hence provides support for using blood cells as a useful accessible tissue for epigenetic biomarker discovery. Our results show that DNA methylation sites in dcSSc patients are enriched for regulatory regions in cell types with key roles in fibrosis, implicating DNA methylation as a modulator of cell functionality. Coupled with the observation that dcSSc and lcSSc are epigenetically distinct disease subtypes, this suggests that the cellular dysfunction observed in dcSSc is, at least partially, due to an epigenetic dysregulation of myeloid cell types. Further, this suggests the possibility of using epigenetic regulation of cell functionality to prevent dysfunction or restore their balance in SSc. Regardless of causality, blood-based biomarkers have the potential to improve risk prediction and help guide treatment decisions. Our findings provide a foundation for further research to determine if the differentially methylated functional loci represent attractive targets for the treatment or prevention of autoimmune- and/or fibrotic-related diseases.

\section{Additional files}

Additional file 1: Figure S1. Visualization of absolute weighted $\beta$ values in whole blood from twin pairs discordant for SSC in the UCSC genome browser showing differential methylation of the IFI44L gene. Fig ure S2. Enrichment of SSC differentially methylated CpGs in regions overlapping histone modifications in the Roadmap Epigenomics Project data. (DOC 536 kb)

Additional file 2: Table S1. Most significant differentially methylated cytosines in whole blood from twins discordant for SSc. Table S2. Most significant canonical pathways, upstream regulators, and diseases and biological functions in differentially methylated genes in whole blood from all twin pairs discordant for SSC. Table S3. Most significant differentially methylated cytosines in whole blood from twins discordant for ICSSC. Table S4. Most significant canonical pathways, upstream regulators, and diseases and biological functions in differentially methylated genes in whole blood from twin pairs discordant for ICSSC. Table S5. Most significant differentially methylated cytosines in whole blood from twins discordant for dcSSc. Table S6. Most significant canonical pathways, upstream regulators, and diseases and biological functions in differentially methylated genes in whole blood from twin pairs discordant for dcSSC. Table S7. Reported SSc-associated gene regions with differentially methylated CpGs in SSc subsets. Table S8. Differentially methylated CpG sites common to this study and to the report by Altorok et al. (2015). Table S9. Cytosines differentially methylated in this study that are also reported as differentially methylated in blood from SLE patients. Table S10. Most significant enrichment of top SSC CpGs overlapping cell type-specific regulatory elements. Table S11. Most significant enrichment of top dcSSc CpGs overlapping cell type-specific regulatory elements. (DOC $913 \mathrm{~kb}$ )

\section{Abbreviations}

dcSSc: Diffuse cutaneous SSc; DHSs: DNase I hypersensitive sites; ICSSC: Limited cutaneous SSC; SLE: Systemic lupus erythematosus; SSc: Systemic sclerosis

\section{Acknowledgements}

The authors would like to acknowledge Debby Hollingshead in the Genomics Research Core at the University of Pittsburgh, and Dr. Timothy Wright for contributing to the recruitment of the twins.

\section{Funding}

This study was supported by the US National Institute of Arthritis and Musculoskeletal and Skin Diseases of the National Institutes of Health (NIH) under Awards Numbers K24 AR060297 (CFB) and K01 AR067280 (PSR), by the Scleroderma Foundation (CFB), private funds (CFB), start-up funds (CFB), and the SmartState and Kitty Trask Holt Endowment (CFB).

\section{Availability of data and materials}

Normalized or raw intensity data of the HM450K BeadChips used during the current study is not yet publicly available because it was generated using private funds, but all data is available upon request from the authors on a collaborative basis.

\section{Authors' contributions}

CFB designed and coordinated the study. CFB and TAM recruited the patients. TAM obtained the history and performed the physical examinations and disease subtyping of patients. CFB processed the blood samples and extracted the DNA. SH confirmed the zygosity of the twins. PSR, KDZ, and $\mathrm{CDL}$ analyzed the data. PSR and CFB wrote the manuscript. All authors were involved in critical review, editing, revision, and approval of the final manuscript. 


\section{Ethics approval and consent to participate}

This study was approved by the Institutional Review Board of the University of Pittsburgh (IRB 0403072). Informed consent was obtained from all participants. All research included in this manuscript conforms with the Declaration of Helsinki.

\section{Consent for publication}

Not applicable.

\section{Competing interests}

The authors declare that they have no competing interests.

\section{Publisher's Note}

Springer Nature remains neutral with regard to jurisdictional claims in published maps and institutional affiliations.

\section{Author details}

'Division of Rheumatology and Immunology, Department of Medicine, Medical University of South Carolina, Charleston, SC, USA. ${ }^{2}$ Department of Public Health Sciences, Medical University of South Carolina, Charleston, SC, USA. ${ }^{3}$ Department of Biostatistics and Data Science, Wake Forest School of Medicine, Winston-Salem, NC, USA. ${ }^{4}$ Center for Public Health Genomics, Wake Forest School of Medicine, Winston-Salem, NC, USA. ${ }^{5}$ Bay Path University, Longmeadow, MA, USA. 'Division of Rheumatology and Clinical Immunology, Department of Medicine, University of Pittsburgh, Pittsburgh, PA, USA.

\section{Received: 27 November 2018 Accepted: 11 March 2019}

\section{Published online: 04 April 2019}

\section{References}

1. Ramos PS, Silver RM, Feghali-Bostwick CA. Genetics of systemic sclerosis: recent advances. Curr Opin Rheumatol. 2015;27:521-9.

2. Feghali-Bostwick C, Medsger TA Jr, Wright TM. Analysis of systemic sclerosis in twins reveals low concordance for disease and high concordance for the presence of antinuclear antibodies. Arthritis Rheum. 2003:48:1956-63.

3. Faulk C, Dolinoy DC. Timing is everything: the when and how of environmentally induced changes in the epigenome of animals. Epigenetics. 2011;6:791-7.

4. Schubeler D. Function and information content of DNA methylation. Nature. 2015:517:321-6.

5. Luo C, Hajkova P, Ecker JR. Dynamic DNA methylation: in the right place at the right time. Science. 2018;361:1336-40.

6. Pai AA, Pritchard JK, Gilad Y. The genetic and mechanistic basis for variation in gene regulation. PLoS Genet. 2015;11:e1004857.

7. Gutierrez-Arcelus M, Lappalainen T, Montgomery SB, Buil A, Ongen H Yurovsky A, Bryois J, Giger T, Romano L, Planchon A, et al. Passive and active DNA methylation and the interplay with genetic variation in gene regulation. eLife. 2013;2:e00523.

8. Pacis A, Tailleux L, Morin AM, Lambourne J, Maclsaac JL, Yotova V, Dumaine A, Danckaert A, Luca F, Grenier JC, et al. Bacterial infection remodels the DNA methylation landscape of human dendritic cells. Genome Res. 2015;25:1801-11.

9. Gutierrez-Arcelus M, Ongen H, Lappalainen T, Montgomery SB, Buil A, Yurovsky A, Bryois J, Padioleau I, Romano L, Planchon A, et al. Tissue-specific effects of genetic and epigenetic variation on gene regulation and splicing. PLoS Genet. 2015;11:e1004958.

10. Teschendorff $A E$, Relton CL. Statistical and integrative system-level analysis of DNA methylation data. Nat Rev Genet. 2018;19:129-47.

11. Ambrosi C, Manzo M, Baubec T. Dynamics and context-dependent roles of DNA methylation. J Mol Biol. 2017;429:1459-75.

12. Javierre BM, Fernandez AF, Richter J, Al-Shahrour F, Martin-Subero الر Rodriguez-Ubreva J, Berdasco M, Fraga MF, O'Hanlon TP, Rider LG, et al. Changes in the pattern of DNA methylation associate with twin discordance in systemic lupus erythematosus. Genome Res. 2010;20:170-9.

13. Lin SY, Hsieh SC, Lin YC, Lee CN, Tsai MH, Lai LC, Chuang EY, Chen PC, Hung CC, Chen LY, et al. A whole genome methylation analysis of systemic lupus erythematosus: hypomethylation of the IL10 and IL1R2 promoters is associated with disease activity. Genes Immun. 2012;13:214-20.

14. Jeffries MA, Dozmorov M, Tang Y, Merrill JT, Wren JD, Sawalha AH. Genomewide DNA methylation patterns in CD4+ T cells from patients with systemic lupus erythematosus. Epigenetics. 2011;6:593-601.
15. Absher DM, Li X, Waite LL, Gibson A, Roberts K, Edberg J, Chatham WW Kimberly RP. Genome-wide DNA methylation analysis of systemic lupus erythematosus reveals persistent hypomethylation of interferon genes and compositional changes to CD4+ T-cell populations. PLoS Genet. 2013;9: e1003678.

16. Zhao M, Liu S, Luo S, Wu H, Tang M, Cheng W, Zhang Q, Zhang P, Yu X, Xia $Y$, et al. DNA methylation and mRNA and microRNA expression of SLE CD4 + T cells correlate with disease phenotype. J Autoimmun. 2014;54:127-36.

17. Coit $P$, Jeffries M, Altorok N, Dozmorov MG, Koelsch KA, Wren JD, Merrill JT, McCune WJ, Sawalha AH. Genome-wide DNA methylation study suggests epigenetic accessibility and transcriptional poising of interferon-regulated genes in naive CD4+ T cells from lupus patients. J Autoimmun. 2013,43:78-84.

18. Webster AP, Plant D, Ecker S, Zufferey F, Bell JT, Feber A, Paul DS, Beck S, Barton A, Williams FMK, et al. Increased DNA methylation variability in rheumatoid arthritis-discordant monozygotic twins. Genome Med. 2018;10:64.

19. van Steenbergen HW, Luijk R, Shoemaker R, Heijmans BT, Huizinga TW, van der Helm-van Mil AH. Differential methylation within the major histocompatibility complex region in rheumatoid arthritis: a replication study. Rheumatology (Oxford). 2014:53:2317-8.

20. Nakano K, Whitaker JW, Boyle DL, Wang W, Firestein GS. DNA methylome signature in rheumatoid arthritis. Ann Rheum Dis. 2013:72:110-7.

21. Liu Y, Aryee MJ, Padyukov L, Fallin MD, Hesselberg E, Runarsson A, Reinius L, Acevedo N, Taub M, Ronninger M, et al. Epigenome-wide association data implicate DNA methylation as an intermediary of genetic risk in rheumatoid arthritis. Nat Biotechnol. 2013;31:142-7.

22. Glossop JR, Emes RD, Nixon NB, Haworth KE, Packham JC, Dawes PT, Fryer AA, Mattey DL, Farrell WE. Genome-wide DNA methylation profiling in rheumatoid arthritis identifies disease-associated methylation changes that are distinct to individual T- and B-lymphocyte populations. Epigenetics. 2014:9:1228-37.

23. de la Rica L, Urquiza JM, Gomez-Cabrero D, Islam AB, Lopez-Bigas N, Tegner J. Toes RE, Ballestar E. Identification of novel markers in rheumatoid arthritis through integrated analysis of DNA methylation and microRNA expression. Autoimmun. 2013:41:6-16.

24. Julia A, Absher D, Lopez-Lasanta M, Palau N, Pluma A, Waite Jones L, Glossop JR, Farrell WE, Myers RM, Marsal S. Epigenome-wide association study of rheumatoid arthritis identifies differentially methylated loci in B cells. Hum Mol Genet. 2017;26:2803-11.

25. Zhu H, Wu LF, Mo XB, Lu X, Tang H, Zhu XW, Xia W, Guo YF, Wang MJ, Zeng $\mathrm{KQ}$ et al. Rheumatoid arthritis-associated DNA methylation sites in peripheral blood mononuclear cells. Ann Rheum Dis. 2019,78:36-42.

26. Ai R, Hammaker D, Boyle DL, Morgan R, Walsh AM, Fan S, Firestein GS, Wang W. Joint-specific DNA methylation and transcriptome signatures in rheumatoid arthritis identify distinct pathogenic processes. Nat Commun. 2016:7:11849.

27. Gervin $K$, Vigeland MD, Mattingsdal M, Hammero M, Nygard $H$, Olsen AO, Brandt I, Harris JR, Undlien DE, Lyle R. DNA methylation and gene expression changes in monozygotic twins discordant for psoriasis: identification of epigenetically dysregulated genes. PLoS Genet. 2012;8: e1002454

28. Park GT, Han J, Park SG, Kim S, Kim TY. DNA methylation analysis of CD4+ T cells in patients with psoriasis. Arch Dermatol Res. 2014;306:259-68.

29. Zhang $P$, Zhao $M$, Liang G, Yin G, Huang D, Su F, Zhai H, Wang L, Su Y, Lu Q. Whole-genome DNA methylation in skin lesions from patients with psoriasis vulgaris. J Autoimmun. 2013;41:17-24.

30. Chandra A, Senapati S, Roy S, Chatterjee G, Chatterjee R. Epigenome-wide DNA methylation regulates cardinal pathological features of psoriasis. Clin Epigenetics. 2018;10:108.

31. Zhou F, Shen C, Xu J, Gao J, Zheng X, Ko R, Dou J, Cheng Y, Zhu C, Xu S, et al. Epigenome-wide association data implicates DNA methylation-mediated genetic risk in psoriasis. Clin Epigenetics. 2016:8:131.

32. Roberson ED, Liu Y, Ryan C, Joyce CE, Duan S, Cao L, Martin A, Liao W, Menter A, Bowcock AM. A subset of methylated CpG sites differentiate psoriatic from normal skin. J Invest Dermatol. 2012;132:583-92.

33. Selmi C, Feghali-Bostwick CA, Lleo A, Lombardi SA, De Santis M, Cavaciocchi F, Zammataro L, Mitchell MM, Lasalle JM, Medsger T Jr, et al. X chromosome gene methylation in peripheral lymphocytes from monozygotic twins discordant for scleroderma. Clin Exp Immunol. 2012; 169:253-62

34. Altorok N, Tsou PS, Coit P, Khanna D, Sawalha AH. Genome-wide DNA methylation analysis in dermal fibroblasts from patients with diffuse and 
limited systemic sclerosis reveals common and subset-specific DNA methylation aberrancies. Ann Rheum Dis. 2015,74:1612-20.

35. Birney E, Smith GD, Greally JM. Epigenome-wide association studies and the interpretation of disease -omics. PLoS Genet. 2016;12:e1006105.

36. Castillo-Fernandez JE, Spector TD, Bell JT. Epigenetics of discordant monozygotic twins: implications for disease. Genome Med. 2014;6:60.

37. LeRoy EC, Medsger TA Jr. Criteria for the classification of early systemic sclerosis. J Rheumatol. 2001;28:1573-6.

38. Morris TJ, Butcher LM, Feber A, Teschendorff AE, Chakravarthy AR, Wojdacz TK, Beck S. ChAMP: 450k Chip Analysis Methylation Pipeline. Bioinformatics. 2014;30:428-30.

39. Willer CJ, Li Y, Abecasis GR. METAL: fast and efficient meta-analysis of genomewide association scans. Bioinformatics. 2010;26:2190-1.

40. Fraga MF, Ballestar E, Paz MF, Ropero S, Setien F, Ballestar ML, Heine-Suner D, Cigudosa JC, Urioste M, Benitez J, et al. Epigenetic differences arise during the lifetime of monozygotic twins. Proc Natl Acad Sci U S A. 2005; 102:10604-9.

41. Bell JT, Tsai PC, Yang TP, Pidsley R, Nisbet J, Glass D, Mangino M, Zhai G, Zhang $F$, Valdes $A$, et al. Epigenome-wide scans identify differentially methylated regions for age and age-related phenotypes in a healthy ageing population. PLoS Genet. 2012;8:e1002629.

42. Horvath S. DNA methylation age of human tissues and cell types. Genome Biol. 2013;14:R115.

43. Breeze CE, Paul DS, van Dongen J, Butcher LM, Ambrose JC, Barrett JE, Lowe R, Rakyan VK, lotchkova V, Frontini M, et al. eFORGE: a tool for identifying cell type-specific signal in epigenomic data. Cell Rep. 2016;17: 2137-50.

44. Weidner Cl, Lin Q, Koch CM, Eisele L, Beier F, Ziegler P, Bauerschlag DO, Jockel KH, Erbel R, Muhleisen TW, et al. Aging of blood can be tracked by DNA methylation changes at just three CpG sites. Genome Biol. 2014;15:R24.

45. Coit P, Yalavarthi S, Ognenovski M, Zhao W, Hasni S, Wren JD, Kaplan MJ, Sawalha AH. Epigenome profiling reveals significant DNA demethylation of interferon signature genes in lupus neutrophils. J Autoimmun. 2015,58:59-66.

46. Coit P, Yalavarthi S, Ognenovski M, Zhao W, Hasni S, Wren JD, Kaplan MJ, Sawalha AH. Epigenome profiling reveals significant DNA demethylation of interferon signature genes in lupus neutrophils. J Autoimmun. 2015;58:59-66.

47. Zhu H, Mi W, Luo H, Chen T, Liu S, Raman I, Zuo X, Li QZ. Whole-genome transcription and DNA methylation analysis of peripheral blood mononuclear cells identified aberrant gene regulation pathways in systemic lupus erythematosus. Arthritis Res Ther. 2016;18:162.

48. Yeung KS, Chung BH, Choufani S, Mok MY, Wong WL, Mak CC, Yang W, Lee PP, Wong WH, Chen YA, et al. Genome-wide DNA methylation analysis of Chinese patients with systemic lupus erythematosus identified hypomethylation in genes related to the type I interferon pathway. PLoS One. 2017;12:e0169553.

49. Grigoryev DN, Mathai SC, Fisher MR, Girgis RE, Zaiman AL, Housten-Harris T, Cheadle C, Gao L, Hummers LK, Champion HC, et al. Identification of candidate genes in scleroderma-related pulmonary arterial hypertension. Transl Res. 2008;151:197-207.

50. Assassi S, Mayes MD, Arnett FC, Gourh P, Agarwal SK, McNearney TA, Chaussabel D, Oommen N, Fischbach M, Shah KR, et al. Systemic sclerosis and lupus: points in an interferon-mediated continuum. Arthritis Rheum. 2010;62:589-98.

51. Christmann RB, Hayes E, Pendergrass S, Padilla C, Farina G, Affandi AJ, Whitfield ML, Farber HW, Lafyatis R. Interferon and alternative activation of monocyte/macrophages in systemic sclerosis-associated pulmonary arterial hypertension. Arthritis Rheum. 2011;63:1718-28.

52. Hudson M, Bernatsky S, Colmegna I, Lora M, Pastinen T, Klein Oros K, Greenwood CMT. Novel insights into systemic autoimmune rheumatic diseases using shared molecular signatures and an integrative analysis. Epigenetics. 2017;12:433-40.

53. Bos CL, van Baarsen LG, Timmer TC, Overbeek MJ, Basoski NM, Rustenburg F, Baggen JM, Thiesen HJ, Dijkmans BA, van der Pouw Kraan TC, et al. Molecular subtypes of systemic sclerosis in association with anti-centromere antibodies and digital ulcers. Genes Immun. 2009;10:210-8.

54. Tan FK, Zhou X, Mayes MD, Gourh P, Guo X, Marcum C, Jin L, Arnett FC Jr. Signatures of differentially regulated interferon gene expression and vasculotrophism in the peripheral blood cells of systemic sclerosis patients. Rheumatology (Oxford). 2006:45:694-702.

55. York MR, Nagai T, Mangini AJ, Lemaire R, van Seventer JM, Lafyatis R. A macrophage marker, Siglec-1, is increased on circulating monocytes in patients with systemic sclerosis and induced by type I interferons and tolllike receptor agonists. Arthritis Rheum. 2007;56:1010-20.

56. Pendergrass SA, Hayes E, Farina G, Lemaire R, Farber HW, Whitfield ML, Lafyatis R. Limited systemic sclerosis patients with pulmonary arterial hypertension show biomarkers of inflammation and vascular injury. PLoS One. 2010;5:e12106

57. Paul DS, Teschendorff AE, Dang MA, Lowe R, Hawa MI, Ecker S, Beyan H, Cunningham S, Fouts AR, Ramelius A, et al. Increased DNA methylation variability in type 1 diabetes across three immune effector cell types. Nat Commun. 2016;7:13555.

58. Rakyan VK, Beyan H, Down TA, Hawa MI, Maslau S, Aden D, Daunay A, Busato F, Mein CA, Manfras B, et al. Identification of type 1 diabetesassociated DNA methylation variable positions that precede disease diagnosis. PLoS Genet. 2011;7:e1002300

59. Horvath S, Raj K. DNA methylation-based biomarkers and the epigenetic clock theory of ageing. Nat Rev Genet. 2018;19:371-84.

60. Zhao M, Zhou Y, Zhu B, Wan M, Jiang T, Tan Q, Liu Y, Jiang J, Luo S, Tan Y, et al. IFI44L promoter methylation as a blood biomarker for systemic lupus erythematosus. Ann Rheum Dis. 2016;75:1998-2006.

61. Imgenberg-Kreuz J, Sandling JK, Almlof JC, Nordlund J, Signer L, Norheim KB, Omdal R, Ronnblom L, Eloranta ML, Syvanen AC, et al. Genome-wide DNA methylation analysis in multiple tissues in primary Sjogren's syndrome reveals regulatory effects at interferon-induced genes. Ann Rheum Dis. 2016;75:2029-36.

62. Altorok N, Coit P, Hughes T, Koelsch KA, Stone DU, Rasmussen A, Radfar L, Scofield RH, Sivils KL, Farris AD, et al. Genome-wide DNA methylation patterns in naive CD4+ T cells from patients with primary Sjogren's syndrome. Arthritis Rheumatol. 2014;66:731-9.

63. Matatiele P, Tikly M, Tarr G, Gulumian M. DNA methylation similarities in genes of black South Africans with systemic lupus erythematosus and systemic sclerosis. J Biomed Sci. 2015;22:34.

64. Suarez-Alvarez B, Rodriguez RM, Schlangen K, Raneros AB, MarquezKisinousky L, Fernandez AF, Diaz-Corte C, Aransay AM, Lopez-Larrea C. Phenotypic characteristics of aged CD4+ CD28null T lymphocytes are determined by changes in the whole-genome DNA methylation pattern. Aging Cell. 2017;16:293-303.

65. Wyman AE, Atamas SP. Sirtuins and accelerated aging in scleroderma. Curr Rheumatol Rep. 2018;20:16.

66. Saito $Y$, Nagae G, Motoi N, Miyauchi E, Ninomiya H, Uehara H, Mun M, Okumura S, Ohyanagi F, Nishio M, et al. Prognostic significance of CpG island methylator phenotype in surgically resected small cell lung carcinoma. Cancer Sci. 2016;107:320-5.

67. Farkas SA, Vymetalkova V, Vodickova L, Vodicka P, Nilsson TK. DNA methylation changes in genes frequently mutated in sporadic colorectal cancer and in the DNA repair and Wnt/beta-catenin signaling pathway genes. Epigenomics. 2014;6:179-91.

68. Valiulyte I, Steponaitis G, Skiriute D, Tamasauskas A, Vaitkiene P. Signal transducer and activator of transcription 3 (STAT3) promoter methylation and expression in pituitary adenoma. BMC Med Genet. 2017;18:72.

69. Men C, Chai H, Song X, Li Y, Du H, Ren Q. Identification of DNA methylation associated gene signatures in endometrial cancer via integrated analysis of DNA methylation and gene expression systematically. J Gynecol Oncol. 2017;28:e83.

70. Leenen FA, Muller CP, Turner JD. DNA methylation: conducting the orchestra from exposure to phenotype? Clin Epigenetics. 2016;8:92.

71. Wynn TA, Vannella KM. Macrophages in tissue repair, regeneration, and fibrosis. Immunity. 2016:44:450-62.

72. Brunasso AM, Massone C. Update on the pathogenesis of Scleroderma: focus on circulating progenitor cells. F1000Research. 2016:5.

73. Lescoat A, Lecureur V, Roussel M, Sunnaram BL, Ballerie A, Coiffier G, Jouneau S, Fardel O, Fest T, Jego P. CD16-positive circulating monocytes and fibrotic manifestations of systemic sclerosis. Clin Rheumatol. 2017,36: 1649-54.

74. Lopez-Cacho JM, Gallardo S, Posada M, Aguerri M, Calzada D, Mayayo T, Gonzalez-Rodriguez ML, Rabasco AM, Lahoz C, Cardaba B. Association of immunological cell profiles with specific clinical phenotypes of scleroderma disease. Biomed Res Int. 2014;2014:148293. 